REMARKS

ON

\section{THE PATHOLOGY OF CHOREA.*}

By W. H. BROADBENT, M.D., M.R.C.P., Senior Assistant-Physician to St. Mary's Hospital and the Fever Hospital.

TIIE cases which I have given are not mere repetitions one of another: almost every one presents some features of interest, and I might have added to them. I have for the moment restricted myself to examples of hemichorea, my immediate object being through them to throw ight on chorea generally; but the more common cases of bilateral chorea offer instructive differences and peculiarities which will reward a careful study.

The first point to be established is, that chorea and hemichorea are essentially similar; and this is clear from the fact that chorea is very commonly unilateral, for a longer or shorter time, before it becomes general; and that, in most cases, the movements predominate on one side or the other throughout. Further, in most cases of hemichorea, if the agitation become severe, it extends in some degree to the limbs of the other side. Examples of unilateral chorea, extending to the limbs of the opposite side when aggravated, are seen in Cases VII and virr. The bilateral action of the muscles of the trunk, eyes, etc., which may be observed in almost all cases of hemichorea, is not what is here spoken of; this will receive consideration further on.

Now unilateral chorea is even less likely to be due to an affection of the spinal cord than unilateral paralysis; and unilateral paralysis from disease of the cord is extremely rare. When we see hemiplegia, we at once refer it to the higher motor centres. It is, however, possible that injury or disease may destroy, to a limited extent, the continuity of one lateral half of the spinal cord, without involving the other, and a few cases of this kind are on record; but, for hemichorea to have its origin in the cord, it would be necessary to suppose that one lateral half of the cord was involved throughout its entire length, from the crura cerebri downwards, without implication of the other-a supposition which is not tenable for a moment. Then, in hemiparaplegia (the name by which Dr. Brown-Séquard calls the cases of disease of one lateral half of the cord), there is motor paralysis on one side of the body below the injured point, and sensory paralysis on the other; whereas in hemichorea, when the sensibility is diminished, it is on the same side with the motor affection (see Cases IV and VII). Taking these facts in connexion with the absence of undue reflex action from tickling, and the considerations advanced by Dr. Russell Reynolds, it seems to me to be demonstrated, not only that the cord is not the special seat of the morbid change which gives rise to chorea, but that it does not participate in the affection, except as the instrument by which the abnormal impulses arising in the higher centres are transmitted to the muscles. An interesting confirmation of this conclusion, and at the same time of the hypothesis which I advanced some time since in explanation of the escape of certain muscles from paralysis in hemiplegia, is found in noting that, in certain parts of the body, the movements of hemichorea transgress the median line, and become more or less bilateral. It is set down in several of the cases given, and it may be observed in almost all cases of hemichorea, that, while the spasmodic muscular contractions are strictly unilateral in the limbs, they are bilateral in the abdomen, chest, and eyes, and imperfectly so in the brow, facial muscles, and tongue. Now these are the very muscles which escape paralysis in hemiplegia; and the theory which explains the one set of facts implies the other. I have shown that the muscles which are not paralysed on the hemiplegic side, in the common form of hemiplegia, are such as habitually act in concert with the corresponding (or some other) muscles of the opposite half of the body, and with difficulty, or not at all, independently of them; and I have attributed their immunity to the fact that, when the muscles of the two sides are thus associated, their nerve-nuclei in the cord are con. nected by commissural fibres, so as to constitute, pro tanto, one common nucleus. We thus have a single nucleus in communication with both corpora striata; and when one corpus striatum is damaged, as in hemiplegia, this common nucleus will still be called into action by the other, and animate the muscles of both sides. On the other hand, when, as in hemichorea, one corpus striatum is discharging irregular

* Concluded from page 347 of last number. impulses, these will affect the common nucleus, and be communicated to both sides.

We come now to another branch of the investigation. Chorea being due to an affection of the sensori-motor ganglia, or of these and the hemispheres, what is the nature of the morbid change?

A preliminary question of some importance has, in the first place, to be decided; i.e., Whether the characteristic symptoms arise out of some peculiarity in the morbid change, or simply out of the functions of the part affected? Whether, in effect, chorea is to be regarded as a symptom or as a disease? To render this point more clear by means of a comparison: The common form of hemiplegia is caused by structural damage of the corpus striatum or thalamus, and this damage may be from hæmorrhage, embolism, softening, tumour, etc. - the paralysis has relation to the seat of the injury, not to its nature. Is chorea similarly symptomatic simply of some abnormal condition of these bodies, which condition may have several causes, or is it indicative of some specific alteration in them? Are the characteristic movements in relation with the seat, or the nature of the morbid change?

This question has only to be put to be deciled. Chorea is symptomatic only of the seat of the disease, and arises out of the function of the part affected. The considerations on which this conclusion is based are as follows. In the first place, it would be an assumption entirely without warrant in pathology to say that the corpora striata and thalami were liable to a morbid process winch never affected the cord or the hemispheres. When, therefore, these latter centres become the seat of the pathological change, which in the corpora striata and thalami give rise to chorea, what are the symptoms produced? And, conversely, what symptoms, having their origin in the sensori-motor ganglia, correspond with delirium as an affection of the hemispheres, or with tetanus as a disease of the cord? Chorea has been called " insanity of the muscles"- - a better phrase would be "delirium of the sensori-motor ganglia." In delirium, there is loss of control over the mental processes, with a rapid production of imperfect ideas; in chorea, loss of control over the motor apparatus, and movements excessive in point of number and extent, but wanting in vigour and precision. All that is aimed at in these remarks, which need not be further pursued, is to show that, just as abolition of function of the hemispheres, sensori-motor ganglia, or cord, may be due to various pathological conditions, and is attended with symptoms characteristic of the part affected, so impairment of function of these centres respectively will be attended with characteristic symptoms, which will be independent of the nature of the cause. We shall not, therefore, expect to find the cause of chorea in any single specific pathological condition of the sensori-motor ganglia. We can fix approximately the range of the morbid change, and obtain some idea of its nature from a consideration of the phenomena of the disease. We can see that the anatomical condition does not amount to actual breach of structure, since that is known to give rise to hemiplegia. It is, however, of a nature to impair the vigour of these ganglia, since the irregular movements are almost always associated with loss of muscular power, and often with diminished sensibility. Chorea is, in fact, next door to paralysis. Cases such as the following, which illustrate this relation, are not uncommon.

CASE X. Partial Hemiplesia passing into Hemichora. - Eliza B., aged 9, came under my care at St. Mary's Hospital May 22, I 865. For three or four weeks she had had considerable loss of power in the right side; could not grasp with the right hand; dragged the right leg; speech indistinct; no facial distortion. Quinine and iron were given, and on June 5th she seemed better. A week later, she was not so well, the weakness in the leg having caused her to fall at times. On June Igth, the hand was stronger, but a jerking was seen in the right side of the body and limbs, and pain was felt in these parts. On July 17 th, she ceased to attend, the agitation having increased. The case had resolved itself into hemichorea.

CASE XI. Fanny C., aged I6, always thin, and, having grown fast, applied as an out-patient on April 6th. She had been losing the use of the right arm and leg for three months; could not hold things in the right hand; dragged the right foot; speech affected at times. Lately, there had been involuntary movements in the right hand occasionally. These movements became choreic. She was ordered three minims of liquor potassæe arsenitis, twenty minims of ammoniated tincture of valerian, and an ounce of infusion of calumba, three times a day; and gradually recovered.

Still more striking is the last of the group of cases given earlier in this paper. There were found, after death, a tumour on the spinal cord, and Addison's disease of the suprarenal capsules; but I venture to conclude that the chorea was not directly due to either of these conditions. In its access, the attack resembled exactly an attack of hemiplegia; and it was, in fact, hemiplegia, plus slight choreic movements; then, as power returned in the affected limbs, the chorea increased, and, for a 
time, was imperfectly bilateral. Had there been no chorea, no one would have hesitated to attribute the hemiplegia to disease of some kind, in or near the corpus striatum. The chorea does not disturb this conclusion; and, obviously, whatever was the cause of the hemiplegia, was the cause of the associated chorea.

The first case narrated in this paper is also interesting in relation to this question. At first, there was unilateral chorea, affecting face, arm, and leg; then, the agitation of the limbs continuing, the face became paralysed to the degree seen in rather severe hemiplegia (this paralysis also having the characters of hemiplegic or centric paralysis, and not of disease or injury of the seventh nerve), all movements ceasing. Again, as the face recovered from the paralysis, the movements reappeared. It is thus evident that in the particular part of corpus striatum in which the facial muscles are chiefly represented, the morbid change caused first impairment, and then temporary abolition of function; and, again, that the morbid condition, which in the corpus striatum as a whole gave rise to chorea, in one particular part, intensified perhaps in some degree, pro. duced paralysis.

We do not get much assistance from post mortem examinations. One reason is, that ceath from chorea is rare; another, more important, is that, from the nature of the disease, the anatomical condition, as has just been pointed out, cannot be one of obvious structural lesion, nor is it certain that it is always of such a character as to be detected by the microscope. Possibly in some instances an alteration of specific gravity may indicate its nature, as in a case mentioned by Dr. Aitken. But a consideration of still greater moment is that, when death occurs in choren, the fatal result is not directly due to the morbid change in the sensori-motor ganglia. Injury of a more severe character, which abolishes their functions, such as laceration, by effused blood, or softening, causing hemiplegia, does not necessarily destroy life. Death in chorea is due either to exhaustion of the strength by the perpetual movements; i.e, it is a secondary result of the local change, and it is not to be accounted for by it; or to disease in some other part of the nervous system, as, for example, in the case related by Dr. Tuckwell in the British and Foreign Medico-Chirurgical Reviea for October 1867, in which there was embolic softening in the hemispheres and in the cord. Possibly, again, death may be caused by other disease entirely foreign to the chorea, or chorea may be simply one symptom among others of a fatal disease, as in a case of typhus fever which $I$ have lately had under my care, in which well-marked chorea came on. It can only lead to error and confusion, then, to fix upon any and every prominent morbid appearance which may be found after death in chorea as the cause of the chorea.

Probalbly the most common and important morbid change giving rise to chorea is capillary embolism in the corpus striatum and thalamus and adjacent parts, the view ably maintained by Dr. Hughlings Jackson. Kirkes in this country, and Sée in France, had pointed out the frequent association of chorea with acute rheumatism; and Kirkes advanced good reasons for the conclusion that the mode of causation was by the detachment of particles of fibrine from the inflamed cardiac valves, and their impaction in the minute vessels of the nervous centres. Dr. Hughlings Jackson has given precision to this view; and it seems to me clear that embolism is, as I have just said, one of the most common and important causes of chorea. If chorea is, as I consider it proved to be, a symptom of some morlid change in the sensori-motor ganglia, and if this change is one which, falling short of structural lesion, is of a nature to impair the functional vigour of these bodies, as is indicated by the accompanying loss of power and impaired sensibility, then no condition more readily fulfils the requirements than obstruction of the minute vessels. But there are reasons which seem to indicate conclusively that it is by no means the only one. It may be admitted-and, indeed, it is clearly proved by numerous observations - that the condition which gives rise to the embolism, the minute cleposits on the valves, may exist without giving rise to a murmur, and that a murmur is often fugitive; but cases are mct with which are not consistent with the hypothesis of embolism. Such are the examples which have been recorded of prompt recovery afier expulsion of intestinal worms, or on the relief of constipation. I have to confess, however, that I have not met with cases of this kind, although continually on the look-out for them, and frequently trying the treatment which would be appropriate. Again, in the chorea of pregnancy, although the condition of blood may be such as to favour the occurrence of embolism, the history does not accord well with this hypothesi:

The different degrees of severity, and the variations in the character of the movements, seem of themselves sufficient to indicate a diversity in the condition of the ganglia. It is scarcely conceivable, for example, that the incessant and violent movements of the worst form of chorea can be due simply to impairment of nutritional vigour in these bodies. Again, there is manifestly some peculiarity in those cases in which the movements persist during sleep, which is not uncommon in the mildest form, and seems to be unusually frequent in hemichorea.

The differences observed in different cases of chorea have not been studied with sufficient care, and no attempt has. been made to interpret them. It would seem that some of them are due to the varying nature of the morbid change; others to the varying extent of the region involved. Variations, probably traceable to differences in the morbid change, are those just mentioned, in which the movements a re extremely violent, and in which they persist during sleep... In the former set of cases, it may be that with embolism there is hyperæmia of vessels not blocked; but this is the merest guess.

We may speak with greater confidence as to the variations due to the extent of the region affected by the morbid change, from sym ptoms indicating impairment of function in other centres. We find, for example, in some cases, sensibility considerably diminished; in others, very slightly. Hemichorea gives us the best opportunity of ascertaining this, as we can use the sound side for comparison. This is explained by supposing the thalamus to be involved in a greater or less degree, as well as the corpus striatum. In other cases again, the intelligence is more obviously impaired-indicating, presumably, implication of the hemispheres; or there may be active delirium or positive mania, as in Dr. Tuckwell's case, already alluded to, in which embolism of the grey matter of the hemispheres was found.

As to the causation of chorea, other than ly embolism, I find it possible to do little more than advance conjectures. Of the conditions which seem to come within the limits prescribed by the symptoms those to which chorea is more commonly attributable are-1, local innutrition; 2, reflex inhibitory influence from peripheral irritation; 3, direct action upon the ganglia by shock, etc.

I am not acquainted with any facts which would warrant the conclusion that inflammation is among the conditions which actually give rise to chorea; and we have evidence to the contrary in the fact that inflam. matory softening, and consequent paralysis, is not preceded or accom. panied by chorea.

It is possible that a morbid condition of the blood may cause chorea, as it may cause delirium, without any change in the central ganglia on the one hand, or hemispheres on the other, appreciable even by means of the microscope. This is the case in typhus very frequently as regards delirium, occasionally as to chorea, an example having been already mentioned. In this instance, the most careful scrutiny failed to detect any morbid alteration in any part of the brain : still, it is not at all probable that chorea, as commonly met with, is thus induced.* As a rule, perhaps, but with many striking exceptions, the children suffering from chorea are pale, thin, and ill-nourished; and it might be supposed that in them the symptoms were traceable to localised exaggeration of the general innutrition in the corpus striatum and thalamus. An organ in a state of relatively great functional activity is likely to feel early and severely the effects of inadequate assimilation of nutritive material; and childhood, the period of life in which chorea occurs, is the period of special activity of the sensori-motor ganglia. The limbs are being educated ; the automatic actions which constitute so large a proportion of their movements are being organised; the associations between eye, ear, hand, and foot, are being formed. Of all these operations, the sensori-motor ganglia are the seat and centre, and their cells and fibres constitute the mechanism of all the varied processes. Not only this, but these ganglia are engaged in forming relations with the hemispnerical ganglia above them. These are receiving and storing up ideas, the basis of which must be sensory impressions, which have been received and transmitted by the thalami, these ideas again becoming the starting point of actions which are formulated, so to speak, by the corpora striata. The higher intellectual functions of the hemispheres are not yet brought into active operation. It can scarcely be doubted that the very great comparative frequency of chorea in childhood stands in some relation with the active functional state of the sensori-motor ganglia during this stage of existence; and this view offers some explanation of the rarity of this affection in infancy, when these centres have not entered upon active work; and in the adult life, when their period of special activity has ceased. Whether mere deficiency of nutritive vigour is sufficient to cause chorea, cannot at present be determined with certainty; but that it may be so, is shewn to be extremely probable, by Dr. C. B. Radcliffe's observations on the relation between debility and irritability, or instability, as the condition indicated may be otherwise characterised.

The ideas commonly entertained about the reaction of peripheral irritation on the nerve-centres are apparently derived from the phenomena of reflex action. An impression on some sentient surface is transmitted

* The discovery by Dr. Bastian of the proximate cause of the delirium of febrile diseases in embolism by altered and cohering white blood-corpuscles, removes almost, if not entirely, the necessity for this vague and unsatisfactory hypothesis. 
to the cord and is reflected to some part of the muscular system, giving rise to motion; it seems natural to conclude that continued irritation will give rise to reppeated movement's, that is, to spasm or convulsion. Nothing seems more simifle or tomprehensilble on this view than chorea caused by the presetice of worms in the intestinal canial, by constipation, by uterine disorders, by pregntancy, etc. "It séems to me that we must admit these conditions as capdbfe of inducing chorea, but the explanation is not so simple. If the movements of chiorea were merely reflex responses to impressions 'made on the intestinal mucotis membrane, their starting point would be the cord, and, as we have seen, the phenomena negative this sipposition. Peripheral ifritation, so far as it is a real cause of chorea, must act, not diretetly but indirectly, by inducing an asthenic condition of the sensori-motor ganglia. The closest parallel would be furnished by examples of reflex paralysis; and the mode of causation may be that by which reflex paralysis is explained, inadequate supply of blood through the action of the sympathetic on the small arteries.

The remaining method in which the nerve-centres may be brought into the state which gives rise to chorea is by the direct effects of shock, such as fright, emotion, etc. I was formerly disposed to rate the influence of fright, so often alleged as a cause of chorea, much lower than I now do. I have met with so many cases in which chorea followed some occasion of alarm, and in which I was unable to trace any other influence which might have induced it, that I cannot 'but conclude that shork is not uncommonly the real cause of this affection. Again, $\mathrm{I}$ harve seen examples of this kind. On the last Guy Fawkes day, a boy; aged five or six, was pursued by a man in a mask. His alarm was such that for a week afterwards he did not cease to tremble; and, from that time, from being a strong healthy boy, with a good colour; he has become weak, pale, and spiritless. A young man came to me after the catastrophe on the ice in Regent's Park; up to that time he had been strong and well, but, after seeing this; although never himself in danger, he was seized with trembling. He could neither eat nor sleep, and became so weak as to be unable to follow his employment, and it was some time before he in any measure recovered his strength. When such effects can be produced on the general health by fear, it is impossible to refuse to believe that chorea may be thus caused. I suppose, too, that cases of insanity, attributed to sudden and violent emotion, are, some of them at least, authentic; and I should look upon chorea in the child as, in some sort, the equivalent of insanity in the adult-a similar affection of a different centre. What the precise character of this affection is, I am not prepared to say.

Although the title of this paper is the Pathology of Chorea, a few words may be said as to the treatment, this being really the end and aim of the investigation. It is not attempted to derive a special line of treatment from the conclusions reached as to the pathology, or even to deduce general principles; but it will be useful to examine, on the one hand, how far these conclusions are supported by the result of experience in the treatment of the disease, and, on the other, to seek for suggestions which may be tested by experiment.

It must be recollected always that chorea, though a disease having a long average duration, tends to a natural recovery; and it will be admitted that the average duration remains long in spite of treatment. This accords perfectly with the views which $I$ have here given, as to the nature of chorea as a disease. If we trace it to a local morbid change, some structural alteration of the corpora striata, and thalami, whether this alteration be due to embolism or not, we shall expect that time will be required for the restoration of the part by the nutritive processes. If the movements were caused by functional irritation of the nerve-centres, whether by the blood or through the nerves, we ought to have far less constancy in duration, and very many more examples of prompt recovery on the removal of an exciting cause.

Not uncommonly in chorea, as in other diseases, the remedy last tried has the credit of a cure due to the completion of a cycle of remedial processes. Not forgetting this fertile source of fallacies, I conclude, from my own experience and from the testimony of others, that the duration of chorea can be shortened by treatment; but, I am equally convinced that the same treatment does not answer in all cases. I have given purgatives, tonics, iron, zinc, cod-liver oil, bromide and iodide of potassium, arsenic, phosphorus, conium, and have had examples of apparent or, I might say, manifest success with most. I have also seen failures with all the heroic methods of administering strychnine till its physiological effects were manifested, and of giving tartar-emetic in enormous doses.

It follows, from the pathology of chorea as understood by me, that different remedies should be required by different cases, chorea being a symptom, not a disease, and arising out of various conditions of the particular nerve-centres of whose disordered function it is the manifestation. The one definite indication derived from pathology is already recognised, i.e., that the disease is one of innutrition. We shall generally do good by giving cod-liver oil and iron, and, perhaps, phosphorus or arsenic. The further object or aim is greater precision in the adaptation of special remedies to particular cases.

\section{THE SOUDAN :}

JOURNEY THROUGH THE DESERT OF CHOB AND THE SOUDAN

PLAINS; WATER-SUPPLY; ITS INFLUENCE ON MALARIOUS AFFECTIONS; PRECAUTIONS TO BE TAKEN AT NIGHT.

\section{BY HENRY BLANC, M.D., M.R.C.S.E., F.R.G.S., etc.,} Staff-Assistant-Surgeon, Bombay Army.

PART III.

ON the I6th of October, 1865 , to our great joy, we at last left Massawah behind us, en route for the highlands, at the very time we had begun to fear, so many had been our deceptions, that to us at least would be demied the privilege of visiting those lands which a few fortunate travellers described as beautiful and strange. For more than a year, with a sighing heart, we had daily gazed upon them as they rose boldly in front of us : a few days journey, a rough but a short march, we knew was quite sufficient to bring us to the very peak of Taranta; and our sorrow was, at first, great indeed when we heard that the Abysinian Emperor had forbidden us to follow that route, and ordered us to enter his dominions by the north-western frontier. Political reasons-not merely a savage fancy-had been the cause that dictated those instructions. Theodore, had he marched towards the north, would have at once scared away the few rebels that had risen in the frontier of Tigré, and the road would have been safe and open; but scarcity prevailed to a great extent in the northern provinces, and, to feed his still large and hungry' host, he had no alternative but to lead them towards the fertile plains of Metsha and Damot; but, at the same time, not in the least disposed to see his hostages fall into the hands of others, he had directed us to cross the Soudan, and enter Abyssinia by the Tschelga Pass, where, as yet, no rebel contested his rule. Four hundred miles, instead of seventy, to him was of little import; but for us, the prospect of a long journey, through malarious plains, at the most unhealthy season of the year, was anything but pleasant. However, as grumbling would have been of no avail, we looked upon the difficulty as cheerfully as we could, and at once set to work to make the best arrangements in our power, so as to secure the health and comfort of the whole party.

We had great trouble in inducing the Bedouins to hire us their camels and accompany us. Their great objection was the unhealthy condition of the country at that time of the year. A month later we could have, in a few days, collected several hundred; and, had it not been for the influence we had acquired over the local authorities, we would not have succeeded, and we would have been obliged to remain several weeks longer at the coast, as, without camels, no start was possible. We had rather a considerable amount of baggage, provisions and stores for six months, and a great many presents for the Emperor himself, or his chiefs. Altogether, we required sixty camels, led by thirty men. Thesc, added to ourselves, the Indian servants, private followers, muleteers, etc., made up our party rather a large one.

The rain in the Soudan had only ceased about a fortnight before; and in those plains, for five or six weeks after the rains, vegetation is so luxuriant, moisture still so considerable, the sun so fearfully hot, the night comparatively so cold, that long experience had taught the natives that, of all months, October is by far the most dangerous to venture in the lowlands. Added to these unfavourable conditions, the quality of the water procurable in that tract of country must be taken into consideration. As a rule, the supply is scanty and bad; the so-called wells are often at a great distance from one another; and the water they contain owes its origin partly to a drainage of the surrounding plains, partly to a small undercurrent generally found in the undersoil of the ravines after the temporary rivers have dried up. The best wells, at least in size, are found in the vicinity of some pastoral tribe, or near some permanent villages; the remainder are little better than muddy pools. The natives have an idea that the fever and other sickness affecting to such a large extent the caravans that cross the Soudan so soon after the rains, are due to the fact that, milk being abundant at such time, the merchants and cameleers drink a great deal of sour milk. So many repeated the same assertion, that I thought there might be some truth in the popular opinion. The only mistake which they make is, that they have not examined the question in its true bearings. Sour milk, I found out from those who indulged in it, gives rise to a strong, painful, and lasting feeling of thirst; the desire for water is so great, that 